\title{
El diseño instruccional ADDIE en la Facultad de Ingeniería de UNIMINUTO
}

\author{
Ilber Darío Saza Garzón ${ }^{1}$ \\ https://orcid.org/0000-0002-3148-7021 \\ Doris Patricia Mora Marín ${ }^{2}$ \\ https://orcid.org/0000-0002-1214-9158 \\ Maryuri Agudelo Franco ${ }^{3}$ \\ https://orcid.org/0000-0002-0233-8676 \\ Corporación Universitaria Minuto de Dios, Colombia
}

The Addie instructional design in the Faculty of Engineering of UNIMINUTO

Recibido: 30-09-2019

Aceptado: 19-12-2019

\section{Cita Recomendada}

Saza, I., Mora, D. \& Agudelo, M. (2019). El diseño instruccional ADDIE en la facultad de ingeniería de UNIMINUTO. Hamut'ay, 6(3), 126-137.

http://dx.doi.org/10.21503/hamu.v6i3.1851

\section{RESUMEN}

El objetivo de esta investigación consistió en Formar a los docentes del departamento de Informática y Electrónica (DIE) de la facultad de ingeniería, en el diseńo y creación de ambientes virtuales de aprendizaje b-Learning, como herramienta de apoyo a los procesos de enseńanza y aprendizaje. En ese sentido las preguntas que orientó este proceso fueron ¿Cuáles son los elementos pedagógicos, didácticos, tecnológicos y evaluativos que requieren los docentes del DIE para la planeación, diseńo, creación e implementación de los ambientes virtuales de aprendizaje (AVA) que aporten al fortalecimiento de las prácticas educativas?, ¿Los profesores están preparados para utilizarlas? Los docentes, que participaron en el proyecto sus edades fueron entre los 35 y 55 años, 12 hombres y 3 mujeres con nivel académico en educación y postgrado

En consecuencia, la metodología de investigación con un componente predominantemente cualitativo, fases: 1 Propuesta de proyecto, 2 Recolección de datos Cualitativo y Cuantitativos, 3 Implementación de las actividades y diseño metodológico, 4 Análisis de datos cualitativos y cuantitativos, 5 Resultados.

Se realizó una recolección de datos en los diferentes talleres y capacitaciones sobre didáctica, herramientas web, tecnologías para el diseño de ambientes virtuales de aprendizaje y sobre todo en el diseño instruccional como herramienta de planeación de los cursos. En consecuencia, este estudio describió el impacto de las capacitaciones en los docentes de la facultad de ingeniería frente a los propios procesos educativos. Los cuales, evidenció mejora en los procesos educativos, debido a la implementación de ambientes virtuales de aprendizaje como herramienta de apoyo tecnológico

\footnotetext{
1 Licenciado en Informática UNIMINUTO, Especialista y Magister en tecnologías de la información aplicadas a la educación De la Universidad Pedagógica Nacional. Docente Universidad Nacional Abierta y a Distancia UNAD. Correo electrónico ilber.saza@unad.edu.co

2 Licenciada en Informática UNIMINUTO y Magister en Educación Universidad Internacional Iberoamericana UNINI. Docente UNIMINUTO. Correo electrónico doris.mora@uniminuto.edu

3 Licenciada en Informática especialista en Diseño de Ambientes de Aprendizaje de UNIMINUTO y Magister en Desarrollo Educativo y Social en Universidad Pedagógica Nacional-Cinde. Correo electrónico maryuri.agudelo@uniminuto.edu
} 
desde el ejercicio de la planeación, aplicación del modelo pedagógico, la didáctica, uso de recursos tecnológicos, elementos propios del diseño instruccional ADDIE.

Palabras Clave: Diseño instruccional, didáctica, formación docente.

\section{Abstract}

The objective of this research was to train teachers from the Department of Informatics and Technology (DIE) of the faculty of Engineering, in the design and creation of b-Learning virtual environments, as a support tool for teaching and learning processes. In that sense, the questions that guided this research were: What are the pedagogical, didactic, technological and evaluative elements that DIE teachers require for the planning, design, creation and implementation of virtual learning environments (AVA) so that they contribute to strengthen educational practices? Are teachers prepared to use them? Teachers, who participated in the project were aged between 35 and 55, among them 12 were men and 3 were women with academic level in education and a postgraduate degree.

Consequently, the research methodology had a predominant qualitative component, with the following phases: 1 Project Proposal, 2 Qualitative and Quantitative data collection, 3 Implementation of the activities and methodological design, 4 Analysis of the qualitative and quantitative data, 5 Results.

Data collection was carried out in the different workshops and training on didactics, web tools, technologies for the design of virtual learning environments and especially in the instructional design as a tool for course planning. Consequently, this study described the impact of training on teachers of the faculty of engineering based on the educational processes themselves. They showed improvement in educational processes, due to the implementation of virtual learning environments as a technological support tool for the planning, application of the pedagogical model, didactics, use of technological resources, proper elements of the ADDIE instructional design.

Keywords: Instructional design, teaching, teacher training.

\section{INTRODUCCIÓN}

En la actualidad en Colombia, en el contexto educativo persiste la implementación de algunas didácticas tradicionales tanto en la escuela como en la educación superior, sin embargo, estas prácticas se llevan a los nuevos escenarios educativos como son los ambientes virtuales de aprendizaje (AVA), quizá porque en muchos casos existe la resistencia a la apertura de prácticas educativas diferentes que van en consonancia con el uso de las tecnologías para la educación, en otros casos por la falta de tecnificación y capacitación docente. Teniendo en cuenta a Echeverry (Estévez, Castro \& Rodríguez 2015), quien afirma existen inconvenientes relacionados con la metodología virtual, motivos de carácter técnico y sociocultural, este último que tiene que ver con el conocimiento sobre las dinámicas de un modelo de formación diferente al presencial y al de distancia tradicional.

En consecuencia, el desconocimiento de cómo hacer e implementar el AVA y las practicas tradiciones aterrizan en los contextos educativos mediados por tecnologías y que van en contra a lo que realmente se espera y en ese sentido los 
docentes desde su espacio de reflexión pedagógica crean interrogantes frente a: ¿Cuál es el proceso para enseñar en espacios virtuales?, ¿Cómo hacer una clase virtual?, ¿De qué manera se establece la comunicación entre docente y estudiante?, ¿La tecnología puede reemplazar al docente?, entre otras.

Ahora bien, las prácticas docentes requieren de cambios en su forma de enseñar, comunicar, evaluar, planear y aprender, y si se adiciona la incursión de las tecnologías digitales en el contexto educativo conlleva a la innovación educativa y pedagógica. Partiendo de lo anterior, en la Corporación Universitaria Minuto de Dios (UNIMINUTO) Sede Principal (S. P.) se evidenció la subutilización de tecnologías digitales y de los espacios de Ambientes Virtuales, estas tecnologías (AVA) son institucionalizadas para el apoyo de los procesos educativos, siendo esto una estrategia de mejoramiento y de calidad educativa, esto se corroboró con una encuesta realizada por los docentes de Gestión Básica de Información (GBI Curso transversal perteneciente a la facultad de ingeniería en UNIMINUTO S. P.) en el año 2016 al Departamento de Informática y Electrónica (DIE), en el cual el 56.14\% de los docentes usan las aulas virtuales, pero de ese porcentaje el 8.9\% usan Objetos Virtuales de Aprendizaje (OVAS), el 10.6\% usan rúbricas de evaluación y el $7.1 \%$ usan diseño Instruccional, resultado que evidencia la falta de utilización de los elementos y componentes que se deben tener en cuenta para el diseño y desarrollo de aulas virtuales.

Teniendo en cuenta este panorama, desde la unidad de Gestión básica de la Información (GBI) que pertenece a la facultad de ingeniería, en el año 2015 se plantea a través de un proyecto de investigación la propuesta de un diseño instruccional en ambientes b-Learning basado en estrategias didácticas y metodológicas que a la luz del enfoque praxeológico y el modelo ADDIE, permitiese optimizar el uso de las aulas virtuales y fortalecer los procesos académicos en dos de los programas de la facultad.

En ese sentido, se decidió continuar con la actividad de mejorar los procesos educativos por medio de la optimización y uso de los recursos tecnoló- gicos con lo que se dispone en UNIMINUTO S. P. y de la aplicación del diseño instruccional del proyecto anteriormente mencionado.

Por lo tanto, en el presente estudio se planteó como objetivo general: Formar a los docentes del Departamento de Informática y Electrónica (DIE) de la facultad de ingeniería, en el diseño y creación de ambientes virtuales de aprendizaje b-Learning como herramienta de apoyo a los procesos de enseñanza y aprendizaje, articulado a un Diseño Instruccional que responde al enfoque pedagógico praxeológico de UNIMINUTO S. P.

Por consiguiente, se trazaron tres objetivos específicos, i. Hacer la caracterización de las habilidades a nivel técnico y pedagógico de AVAS, ii. Capacitar a los miembros de la comunidad docente en estos aspectos, y iii. Reconocer el impacto de la experiencia en la implementación de lo aprendido de los AVA y el proceso educativo y labor docente.

\section{Ambientes Virtuales de Aprendizaje. (AVA)}

Con la invención de la tecnología digital, la cual incursionó en los diferentes contextos sociales, (económico, social, cultural, antropológico, educativo, etc.), generando cambios disruptivos; en el aspecto educativo, hoy en día es imposible pensar hacer una consulta o crear un documento escrito sin el computador e Internet, en consecuencia, se han venido creando nuevos espacios educativos y cambiando los existentes.

Por ende, la educación tradicional se vio en la necesidad de crear asignaturas como la informática, como respuesta a las necesidades de saber usar y comprender las dinámicas sociales con la incursión de la tecnología. Además, se empezó a implementar tecnologías como la televisión, videos, computadores en las aulas, como herramienta de apoyo a los procesos de enseñanza y aprendizaje.

En cuanto a la educación a distancia, fue la principal beneficiada, porque el computador e internet cambio las practicas educativas de forma radical, dejando a un lado los correos certificados, validación por radio y televisión (en el contexto colombiano) por la implementación de Plataformas de 
gestión y administración del conocimiento (LMS y CMS) o lo que se llama Ambientes Virtuales de Aprendizaje. A propósito del término Coll \& Monereo, (2008) Explica que este concepto de AVA, el cual consiste como el proceso virtual en donde se involucra diferentes organizaciones y comunidades, en este contexto realiza sus actividades y prácticas; así como generar espacios de comunicación entre los diferentes actores que conforman estas comunidades.

Ahora bien, en UNIMINUTO como estrategia de mejora continua al proceso educativo, estableció el uso de AVA como estrategia de calidad educativa, en acompañamiento y uso efectivos de los recursos tecnológicos en las asignaturas de los diferentes programas académicos nivel presencial, es decir, el uso de ambientes blender learning, (uso de AVA en cursos presenciales).

\section{Diseño Instruccional (DI)}

Tiene su origen y componente conductual, el cual se puede interpretar como una serie de instrucciones a seguir, pero en este punto se desea enfocar como una guía para la planeación, diseño y construcción de un ambiente virtual de aprendizaje, mas no en la propuesta pedagógica y en implementación; el DI parte de la reflexión de cómo mejorar las prácticas docentes desde una perspectiva didáctica y tecnológica para que el interés de los estudiantes sea mejor. Por lo tanto, el DI está relacionado al mejoramiento y rendimiento de los procesos educativos en el que convergen diferentes esfuerzos profesionales (docentes, diseñadores gráficos, pedagogos expertos en determinado saber, etc.), institucionales (universidades, colegios, institutos, etc.), gubernamentales, que buscan ofrecer educación de calidad, respondiendo a las necesidades y demandas de un mundo globalizado, en un esfuerzo conjunto por alcanzar la eficiencia y eficacia, permitiendo que esta llegue a todos con la misma calidad (Pérez, López \& Peralta, 2004).

Ahora bien, DI es un proceso en el cual se analizan, diseñan, organizan y presentan los contenidos con sentido pedagógico; este incluye las estrategias de enseñanza-aprendizaje necesarias para ser aplicadas en entornos virtuales. Según
Williams et al., (s.f) dicen que el diseño instruccional la entiende como un ejercicio pragmático, que se fundamenta desde la teoría de aprendizaje con el fin de producir una formación eficaz, por ende, el DI forma parte del contenido educativo digital, ofreciendo contenidos, estructuración, forma de comunicación, evaluación, seguimiento didácticas y pedagogías que generan experiencias satisfactorias de aprendizaje en el estudiante.

Para Velarde et al., (2017) el diseño instruccional tiene pasos a seguir, planificar y ordenar, fundamentado en las teorías del aprendizaje, (p. 76). En esa concordancia el DI puede ser desde diversas teorías de aprendizaje como la tradicional, conductual, cognitiva y constructivista.

Por consiguiente, el Diseñador Instruccional plantea estrategias metodológicas según las necesidades académicas y del contexto inmediato facilitando el proceso de aprendizaje; estas estrategias parten de teorías como la asimilación, aprendizaje colaborativo y cooperativo, diagramas, aprendizajes por descubrimiento, Aprendizaje Basado en Problemas, aprendizaje significativo, teorías de elaboración entre otros.

Otro elemento que debe tener el DI es la dimensión tecnológica, en donde se selecciona herramientas digitales (cmapcloud, drive, powtoon, prezi, genially, emaze, entro otros) y recursos; cabe aclarar que es necesario hacer la reflexión pedagógica ya que el DI deben obedecer a las necesidades educativas y situaciones del contexto, por lo tanto, la teoría de aprendizaje, estrategias didácticas y recursos logísticas y económicos corresponden a los requerimientos institucionales, educativos y del estudiante.

El DI representa el puente, la conexión entre las teorías del aprendizaje, las didácticas, modelo pedagógico, recursos tecnológicos, forma de evaluación y su puesta en práctica. (Benítez, 2010).

Por otra parte, existen diferentes DI cada uno con diversas formas de planear y diseñar un AVA, cada uno de ellos presenta características diferentes, sin embargo, en esta propuesta se centra en el diseño tipo ADDIE.

El DI tipo ADDIE fue seleccionado para las aulas virtuales de UNIMINUTO de la facultad de 
ingeniería debido a su facilidad, flexibilidad y coherencia con el enfoque pedagógico de la universidad.

Ahora bien, el modelo ADDIE (Figura 1) es una sigla que representa distintos momentos de un curso virtual, según Belloch (2017) explica que el modelo ADDIE es un modelo interactivo no lineal, debido a que la evaluación formativa que se ejecuta en cada fase permite al diseñador instruccional el poder hacer y ejecutar cambios.

\section{Propuesta Modelo ADDIE}

La ejecución y desarrollo de las etapas iniciales fueron insumo vital para la consolidación del diagnóstico como línea de partida, en la que se posiciona la necesidad del docente de la facultad de ingeniería de UNIMINUTO S. P. por fortalecer y desarrollar competencias en el diseño de ambientes virtuales de aprendizaje, elección de un adecuado diseńo instruccional, reconocimiento y aplicación de herramientas TIC y web 2.0 y por último buen uso de los recursos disponibles en la plataforma Moodle; en respuesta a ello se plantea la propuesta del diseño instruccional basado en el modelo de ADDIE y principios del enfoque praxeológico, donde se propone al docente de UNIMINUTO realizar su propio Guión instruccional y Guía didáctica para la posterior implementación en el aula.

Por lo anterior, partiendo del Diseño Instruccional desarrollado en la fase anterior, se decide implementar una propuesta de capacitación en ambientes virtuales de aprendizaje cuyo objetivo principal es "Formar a los docentes del Departamento de Informática y Electrónica (DIE) de la facultad de ingeniería, en el diseño y creación de ambientes virtuales de aprendizaje b-Learning como herramienta de apoyo a los procesos de enseñanza y aprendizaje". En ese sentido, se crea e implementa un curso virtual con una duración en su ejecución de 60 horas.

La fase de Análisis es la primera fase que permite identificar las necesidades académicas, logísticas, tecnológicas educativas, conocer el contexto y lo que se requiere, en este momento se postula los objetivos de aprendizaje las metas, y se organiza el currículo.

La fase del Diseño consiste en establecer la propuesta pedagógica por medio de la teoría de aprendizaje, en el caso de UNIMINUTO el enfoque praxeológico, con ello se organizan las estrategias didácticas, entre las cuales están las metodologías que facilitan el aprendizaje (Webquest, Aprendizaje ABP, estudio de casos entre otros) y los procesos de evaluación. Según Muñoz \& González (2009) exponen que el Diseño implica hacer un análisis del contexto aplicar esos resultados, con el fin de diseñar estrategias para el diseño, y desarrollo de las diferentes instrucciones. Es decir que, durante esta fase, se postula las metas de aprendizaje y los objetivos a alcanzar (p. 17).

En el Desarrollo, se crean los distintos recursos que están en concordancia con el currículo, los objetivos y la propuesta pedagógica, como son las presentaciones, vídeos, animaciones, PDF, simuladores entre otros objetos virtuales de aprendizaje (OVA), todos estos elementos están previamente definidos en el formato del DI creado en la fase anterior y se implementan en el ambiente virtual de aprendizaje alojado en la plataforma Moodle.

La fase de Implementación es la puesta en marcha del ambiente virtual de aprendizaje, se aplica la planeación, recursos, currículo y sobre todo la propuesta pedagógica. Además, la implementación permite revisar la efectividad y eficacia de los elementos dispuesto en el AVA.

La fase de Evaluación donde se debe medir y hacer seguimiento constante y permanente a cada una de las etapas anteriores, en este punto se busca conocer la eficacia y eficiencia de los desempeños en el estudiante, docente, recursos tecnológicos, currículo y propuesta pedagógica, en otras palabras, esta etapa consiste en hacer la revisión del AVA con el fin de identificar qué aspectos son necesarios reforzar, cambiar y mejorar, para generar cambios que permitan trascender las realidades. Según Sánchez et al., (2018) postulan que la evaluación debe ser formativa y sumativa, la primera durante todo el proceso y en cada una de las fases y sumativa cuando se termina el proceso educativo. 


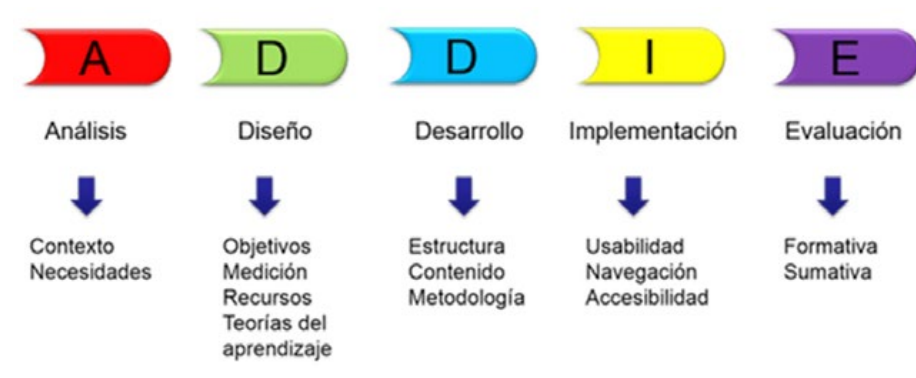

Figura 1. Modelo ADDIE.

Fuente: Elaboración propia (Diseño basado en Williams, et al., 2004)

Por lo tanto, la evaluación según el modelo ADDIE no es el fin, sino que se debe aplicar a lo largo de todo el proceso, por ello Morales, Edel \& Aguirre (2014) afirman que la evaluación permite medir la calidad de los procesos educativos, tanto de planeación, diseño como ejecución e implementación. Así mismo, Centeno (2017). Postula que, en esta fase la evaluación debe ser formativa y sumativa, donde el ejercicio requiere ser realizado desde el inicio, diseño, creación e implementación de los resultados, los elementos tecnológicos y pedagógicos que involucran el ejercicio educativo.

En consecuencia, el DI y en específico el ADDIE es un ejercicio de planeación riguroso que requiere de elementos pedagógicos, tecnológicos, organizativos y evaluativos.

\section{Propuesta de Modelo Pedagógico Praxeológico}

La Praxeología consiste en un ejercicio de reflexión frente a la práctica educativa, en palabras de Juliao (2011) este enfoque se entiende como "un discurso (logos) construido después de una seria reflexión, sobre una práctica particular y significante (praxis); como un procedimiento de objetivación de la acción, como una teoría de la acción" (p. 27), lo anterior quiere decir que, tanto docentes como estudiantes están en constante reflexión y cambio frente a sus acciones educativas, con el fin de llegar a la mejora continua.

Desde esta perspectiva en UNIMINUTO se estableció la Praxeología como el enfoque pedagógico, que obedece a la reconfiguración del sujeto social desde su formación profesional, por tanto, el modelo pedagógico praxeológico se centra en la formación integral permite que el docente incluya componentes y elementos en su praxis que trascienden su quehacer docente, generando procesos reflexivos desde la eficacia, la pertinencia y la responsabilidad de sus acciones. Con el propósito que el docente potencie su ejercicio, se llevó a cabo esta propuesta, que requirió de un estudio y análisis del enfoque Praxeológico que permita al docente hacerla parte de su ejercicio pedagógico, por lo tanto, para esta investigación se hizo necesario una revisión minuciosa que permitió relacionar el enfoque pedagógico propio de UNIMINUTO y el Diseño Instruccional ADDIE.

Cabe mencionar que, UNIMINUTO propende por la proyección y desarrollo social, es decir que los profesionales posibilitan espacios de construcción social equitativa y justa desde su saber, por lo tanto, los estudiantes son competentes en su actuar, así como en crear espacios para la reflexión sobre las acciones que realiza frente a la responsabilidad eficacia en el aprendizaje y ejecución del mismo, con el fin de aprender de las vivencias para así mejorar sus experiencias.

Es por ello por lo que, desde la reflexión de la praxis, el enfoque praxeológico presenta cuatro fases que no necesariamente son en el presente orden, ya que este es cíclico y se puede dar en el orden que se desee, según las circunstancias del acto educativo, estas fases son:

- El ver, consiste en analizar el contexto, identificar las necesidades educativas a nivel curricular, pedagógico, logístico entre otros elementos, si se plasma en el DI ADDIE tiene una profunda relación entre estos dos elementos, ya que en ambas fases se parte del reconocimiento de la situación actual. Según Juliao (2002) menciona que "El observador requiere una mirada atenta y de escucha, lo cual no proviene solo de un procedimiento de reflexión sino, ante todo, de la indagación de la practica viva" (p. 101)

- El juzgar, es el proceso donde se analiza el contexto, se hace críticas frente a las distintas situaciones observadas, generando indagación y estudio sobre diversas teorías para comprender la práctica crear estrategias y planes de acción, de este modo tener un punto de vista propio. 
En otras palabras ¿Qué cosas son las que se deben cambiar?, en el DI ADDIE se ubica en el diseńo, donde se plantea los objetivos de aprendizaje, los elementos pedagógicos y metodológicos.

- El actuar, que lleva a generar un plan de acción, por lo que se realizan las guías, recursos, objetos virtuales de aprendizaje, se implementan las didácticas y estrategias pedagógicas, en otras palabras, es la etapa del DI ADDIE, donde se diseña e implementa al ambiente virtual de aprendizaje.

- Devolución creativa, cosiste en un proceso de auto reflexión sobre los resultados obtenidos, en este ejercicio se analiza y evalúa las cosas que deben seguir, las que deben mejorar o las que debe cambiar, gracias a ese proceso de evaluación y autorreflexión de cada una de las acciones y procesos realizados, para lograr cambios y mejoras, en contraste con el DI ADDIE se relaciona con el proceso de evaluación.

A partir de lo anterior, se evidencia la relación entre la postura Praxeológica y el modelo del diseño ADDIE, dando cuenta de la concordancia desde el ejercicio práctico, organizativo y de evaluación del AVA.

De esta manera los docentes participaron de una serie de capacitaciones, entre las cuales implicó el reconocimiento de las fases y apropiaron la relación del diseño ADDIE con el enfoque Praxeológico, este proceso de aprendizaje fue acompañado por medio de recursos como documentos, videos y talleres, que permitieron una fácil apropiación, con el fin de generar cambios en las prácticas docentes y reflexiones desde la importancia de la fundamentación pedagógica que deben tener los AVA.

En cuanto a la capacitación a los docentes de la facultad de ingeniería hubo un tema que fue la apropiación tecnológica, este se dividió en dos ítems, el primero desde las plataformas para AVA y el segundo en el uso de tecnologías Web.

\section{Materiales y Métodos}

\section{Participantes}

La muestra estuvo compuesta por los docentes de la facultad de ingeniería de UNIMINUTO, son profesionales expertos en su saber disciplinar, el $90 \%$ presentan posgrados a nivel de especialización y maestría, un $5 \%$ cuenta con doctorado, el $20 \%$ de la población ha realizado curso en pedagogía o tiene especialización y/o maestría en educación.

La población de estudio en la facultad de ingeniería es de 180 profesores de planta (docente tiempo completo, medio tiempo y hora catedra), no fue posible contar con toda la población, debido a los tiempos que se disponían por ende, se realizó una muestra no probabilística accidental, (Otzen \& Manterola, 2017), debido a que se consideró los tiempos del que disponían, en ese sentido se contó con 15 docentes para la ejecución de las capacitaciones, diseño y creación de ambientes virtuales de aprendizaje con el sentido pedagógico.

Los docentes, que participaron en el proyecto se caracterizan por aprender y mejorar sus practicas pedagógicas, oscilan en edades entre los 35 y 55 ańos, de los cuales hubo 12 hombres y 3 mujeres con nivel académico en educación profesional y postgrado (como política UNIMINUTO para contratación). Al no formar parte del análisis de la investigación no se recolectó datos sociodemográficos de los participantes.

\section{Instrumento}

En cuanto a los instrumentos de recolección de datos cualitativos se realizó la observación in situ por medio del seguimiento de los docentes en la participación de los diferentes foros (foro de componente pedagógico, recursos tecnológicos, reflexión pedagógica y evaluación del curso), estas participaciones permitieron identificar el nivel de apropiación de los temas tratados.

Otro instrumento de recolección fue el seguimiento a las actividades y trabajos realizados y la forma cómo los aplicaron en el formato de DI (Figura 2) y en consecuencia el desarrollo de esta 
planeación en las aulas virtuales, evidenciando el nivel de articulación del DI diligenciado con el AVA.

TEMA 2: Desarrollo y aplicación de contenidos en la WEB 2.0

\begin{tabular}{|c|c|c|c|c|}
\hline \multicolumn{5}{|c|}{$\begin{array}{l}\text { OBJETIVOS: Desarrollar contenidos de la Web } 2.0 \text { y aplicarlos a su proyecto de Aula, donde fundamentalmente } \\
\text { se orienten a proyección social. }\end{array}$} \\
\hline \multicolumn{5}{|c|}{$\begin{array}{l}\text { COMPETENCIAS: Crear conciencia de la importancia del manojo de las herramientas Web } 2.0 \text { y su orientación } \\
\text { hacia su compromiso social }\end{array}$} \\
\hline \multirow[b]{2}{*}{ Sub tema 1} & \multirow[b]{2}{*}{ Contenido } & \multirow[b]{2}{*}{ Fases } & Recursos & \multirow[b]{2}{*}{ Bibliografia } \\
\hline & & & \begin{tabular}{|l|l|l|l|l|l|}
$E$ & $T$ & $V$ & $P$ & $F$ & $A$
\end{tabular} & \\
\hline \multirow{6}{*}{$\begin{array}{l}\text { Pertinencia } \\
\text { y usos en } \\
\text { las presen- } \\
\text { taciones } \\
\text { gráficas } \\
\text { online }\end{array}$} & \multirow{6}{*}{$\begin{array}{l}\text { - Generalidades } \\
\text { en el diseño de } \\
\text { presentaciones } \\
\text { - Caracteristicas } \\
\text { de las } \\
\text { presentaciones } \\
\text { online (Prezi) }\end{array}$} & Ver: Identifica las diferentes posibilidades de & & \multirow{4}{*}{$\begin{array}{l}\text { Planeta Web } 2.0 \\
\text { Inteligencia Co- } \\
\text { lectiva o medios } \\
\text { FastFood; } \\
\text { Cristóbal Cobo } \\
\text { Roma ni /Hugo } \\
\text { Pardo }\end{array}$} \\
\hline & & $\begin{array}{l}\text { (www.prezi.com) teniendo como referente un tema } \\
\text { relaclonado con su propuesta de investigación. }\end{array}$ & & \\
\hline & & Juzgar: Utiliza las herramientas de la Web 2.0, como & & \\
\hline & & $\begin{array}{l}\text { recurso académico para la administración de la } \\
\text { información basados en los espacios virtuales que el } \\
\text { mundo de la Informática proporciona actualmente. }\end{array}$ & Otros: & \\
\hline & & Actuar: En la medida en que un estudlante alcance y & 口 & \\
\hline & & $\begin{array}{l}\text { desarrolle su escala de valores, podrá alcanzar en } \\
\text { mayor medida su formación profesional; lo } \\
\text { verdaderamente Importante consiste en afianzar } \\
\text { aquellos valores que por excelencia establezcan las } \\
\text { bases de un ser humano integro, con capacidad } \\
\text { para separar lo bueno de lo malo, para instaurar } \\
\text { procesos "puros", serios, responsables, con } \\
\text { fortalezas para ambientar conductas claras y } \\
\text { honradas donde la Información sea el eje principal y } \\
\text { el ser humano su manipulador primario. } \\
\text { Según lo anterior se proponen como el sistema de } \\
\text { valores del curso: } \\
\text { - Etica y Responsabilidad en el manejo de la } \\
\text { información. Espiritu colaborativo y de servicio } \\
\text { referente a la recepción y distribución de la } \\
\text { información. } \\
\text { - Respeto enmarcado en el desarrollo de } \\
\text { actividades donde la Información actúe como } \\
\text { objeto principal. } \\
\text { - La verdad como filosofia interna de la información. } \\
\text { - La moral, dada desde lo más interno del ser y } \\
\text { proyecta a la realización de actividades y procesos } \\
\text { a través de medios }\end{array}$ & Otros: & \\
\hline
\end{tabular}

Figura 2. Formato de Diseño instruccional en Modelo ADDIE y enfoque Praxeológico.

Fuente: Elaboración propia (2017)

Los instrumentos de recolección de datos cuantitativos, se realizó una encuesta previa al curso de capacitación, para identificar el nivel de apropiación de los recursos tecnológicos, conocimiento de DI y del enfoque pedagógico de la universidad, estos datos dieron pautas para la organización del aula virtual, conocer las necesidades curriculares, pedagógicos y tecnológicas para implementar la capacitación, posteriormente se usó toma de datos cuantitativos para conocer el nivel de interacción de los usuarios del aula en los temas, simuladores, tutoriales, lecturas, y demás recursos dispuesto en la capacitación.

\section{Tipo y Diseño}

El tipo de estudio es explicativo y el diseño utilizado se planteó desde una investigación mixta, donde convergen los datos cualitativos y cuantitativos. (Campos 2009):

Un estudio de método mixto incluye la reco- lección y análisis de datos tanto cualitativos como cuantitativos en un solo estudio, donde se recogen concurrente o consecuencialmente, se dan según cierta prioridad o dominancia. Incluye, asimismo, la integración de los datos en una o más etapas del proceso de investigación, (p. 34).

En esta investigación se presenta un componente cualitativo en la observación y estudio del plan curricular y la implementación de la capacitación a través de un ambiente virtual de aprendizaje e-Learning para los docentes de DIE de la facultad de ingeniería, en la que se busca conocer la incidencia del curso frente a los procesos de enseñanza que aplican los docentes, además de identificar la eficacia, pertinencia del DI, tanto para el curso de capacitación como para la aplicación del mismo en las aulas virtuales creadas por los docentes capacitados.

Por otra parte, un componente cuantitativo, debido a que los números y la estadística permite categorizar e identificar qué competencias son necesarias desarrollar en los docentes, lo anterior se logró por medio de una encuesta antes del curso para reconocer las habilidades de los docentes frente a DI y AVA, del análisis de estos datos se pudo definir los elementos curriculares de cursos de capacitación, además los datos numéricos permiten conocer el nivel de aprobación del curso de capacitación teniendo en cuenta los elementos pedagógicos, tecnológicos y curriculares.

Al final del curso cada docente que participó en la capacitación entregó un curso virtual como herramienta de apoyo a una materia para el periodo 2017/2.

En consecuencia, el diseño metodológico de la investigación es de carácter Exploratorio secuencial, en donde la investigación inicia con la toma de datos cualitativos explicar aquellos fenómenos que los números no pueden hacer.

Según López \& Fachelli. (2015) la convergencia entre la toma de datos cualitativos y cuantitativos debido a su forma de interpretar y analizar se muestra en la Figura 3. 


Selección Conceptual
Variables e Indicadores $\rightarrow \begin{aligned} & \text { Realización de } \\ & \text { encuesta }\end{aligned} \Rightarrow \begin{aligned} & \text { Análisis cuantitativo } \\ & \text { algebraico, estadistica }\end{aligned}$

Figura 3. De lo cualitativo a lo cuantitativo. López \& Fachelli. (2015)

Además, refiere que este proceso implica que los datos cualitativos nutren y complementan los datos cuantitativos, esto facilita la transformación y reducción en los términos, categorías para crear los análisis.

\section{Procedimiento}

Partiendo de la metodología y diseño investigativo se planteó las siguientes actividades. (Tabla 1)

\section{Tabla 1}

Diseño y fases de la propuesta metodológica

\begin{tabular}{|c|c|c|}
\hline Fase & Actividad & Resultados \\
\hline $\begin{array}{l}\text { Fase } 1 \\
\text { Propuesta de } \\
\text { proyecto: }\end{array}$ & $\begin{array}{l}\text { Creación del documento } \\
\text { de investigación, entrega } \\
\text { del mismo según el formato } \\
\text { establecido para la convo- } \\
\text { catoria }\end{array}$ & $\begin{array}{l}\text { Documento } \\
\text { de investiga- } \\
\text { ción }\end{array}$ \\
\hline $\begin{array}{l}\text { Fase } 2 \\
\text { Recolección } \\
\text { de datos } \\
\text { Cualitativo y } \\
\text { Cuantitativos }\end{array}$ & $\begin{array}{l}\text { En esta etapa se decide } \\
\text { el tipo de investigación y } \\
\text { metodología, teniendo en } \\
\text { cuenta la naturaleza del } \\
\text { documento de investiga- } \\
\text { ción, en ese orden de ideas } \\
\text { se propone investigación } \\
\text { mixta, siendo un diseño } \\
\text { Exploratorio secuencial. } \\
\text { Por lo tanto, se postula los } \\
\text { diferentes instrumentos } \\
\text { de recolección de datos } \\
\text { cualitativos a través de } \\
\text { entrevista semi-estructu- } \\
\text { rada y observación directa } \\
\text { de los diferentes procesos } \\
\text { de capacitación, diseño, } \\
\text { implementación de las } \\
\text { aulas virtuales, así como } \\
\text { los diferentes procesos de } \\
\text { enseñanza. }\end{array}$ & $\begin{array}{l}\text { Capacita- } \\
\text { ciones a } \\
\text { docentes en } \\
\text { los elementos } \\
\text { pedagógicos } \\
\text { y tecnológicos }\end{array}$ \\
\hline $\begin{array}{l}\text { Fase } 3 \\
\text { Implementación } \\
\text { de las activida- } \\
\text { des y diseño } \\
\text { metodológico }\end{array}$ & $\begin{array}{l}\text { En esta etapa se desarrolla- } \\
\text { rán las actividades propues- } \\
\text { tas para este proyecto entre } \\
\text { las cuales esta: } \\
\text { - Caracterización docente } \\
\text { del DIE. }\end{array}$ & \\
\hline
\end{tabular}

\begin{tabular}{|c|c|c|}
\hline Fase & Actividad & Resultados \\
\hline & $\begin{array}{l}\text { - Identificación de las ne- } \\
\text { cesidades pedagógicas y } \\
\text { tecnológicas. } \\
\text { - Capacitaciones y segui- } \\
\text { miento. } \\
\text { - Diseño y desarrolla del } \\
\text { aula virtual del DIE. } \\
\text { - Observación de la imple- } \\
\text { mentación de las aulas } \\
\text { del DIE. } \\
\text { - Toma de datos estudian- } \\
\text { tes frente al impacto de } \\
\text { las aulas virtuales. }\end{array}$ & $\begin{array}{l}\text { Capacita- } \\
\text { ciones a } \\
\text { docentes en } \\
\text { los elementos } \\
\text { pedagógicos } \\
\text { y tecnológicos }\end{array}$ \\
\hline $\begin{array}{l}\text { Fase } 4 \\
\text { Análisis de da- } \\
\text { tos cualitativos } \\
\text { y cuantitativos: }\end{array}$ & $\begin{array}{l}\text { Teniendo en cuenta los } \\
\text { datos recopilados se } \\
\text { realizará el análisis de los } \\
\text { datos numéricos y cómo } \\
\text { estos pueden ser interpreta- } \\
\text { dos y entendidos desde los } \\
\text { datos cualitativos, para ello } \\
\text { la metodología a trabajar } \\
\text { es la triangulación el cual } \\
\text { consiste en tomar las } \\
\text { conclusiones de los datos } \\
\text { y cruzarlos con teorías y } \\
\text { planteamientos de expertos } \\
\text { en el tema que puedan } \\
\text { validar la información. Por } \\
\text { otra parte, este análisis de } \\
\text { datos nos permite empezar } \\
\text { a hacer la reflexión respecto } \\
\text { a qué acciones se pueden } \\
\text { formular para apoyar los } \\
\text { procesos formativos de los } \\
\text { docentes del DIE. }\end{array}$ & $\begin{array}{l}\text { - Análisis } \\
\text { de datos } \\
\text { recogidos } \\
\text { en la imple- } \\
\text { mentación } \\
\text { y desarrollo } \\
\text { del proyec- } \\
\text { to, estos } \\
\text { datos son } \\
\text { de corte } \\
\text { Cualitativo } \\
\text { y cuantita- } \\
\text { tivos }\end{array}$ \\
\hline $\begin{array}{l}\text { Fase } 5 \\
\text { Resultados: }\end{array}$ & $\begin{array}{l}\text { Para finalizar, esta última } \\
\text { etapa trata de realizar el in- } \\
\text { forme final sobre el proceso } \\
\text { de caracterización de los } \\
\text { procesos pedagógicos, di- } \\
\text { dácticos y tecnológicos que } \\
\text { llevan a cabo los docentes } \\
\text { de DIE en la implementación } \\
\text { de las aulas virtuales y pos- } \\
\text { tular posibles soluciones (si } \\
\text { se requiere) para aportar en } \\
\text { la formación y mejora conti- } \\
\text { nua de la Facultad y del de- } \\
\text { partamento de Informática y } \\
\text { electrónica. }\end{array}$ & $\begin{array}{l}\text { Entrega de } \\
\text { las aulas } \\
\text { virtuales im- } \\
\text { plementadas } \\
\text { por los docen- } \\
\text { tes seleccio- } \\
\text { nados para } \\
\text { participar en } \\
\text { este proyecto. }\end{array}$ \\
\hline
\end{tabular}

Fuente: Elaboración propia (2017). 


\section{Resultados}

El DI ADDIE cumple con las necesidades educativas de los docentes y estudiantes de UNIMINUTO, puesto que, le permite crear espacios de reflexión frente a las didácticas y recursos a implementar, en un AVA el cual repercute en los espacios presenciales.

Los docentes que recibieron la capacitación combinaron diferentes metodologías y didácticas apoyadas por tecnologías como fue la creación de sitios Web con propósito pedagógicos como Webquest, Wikis, situaciones ABP o estudio de caso, estas páginas Web se incrustaron en moodle por medio del módulo etiqueta.

En cuanto al segundo ítem, tecnologías Web, por medio de simuladores y videotutoriales los docentes reconocieron algunas de estas herramientas y la aplicación en los AVA, por lo que se categorizaron según su aplicabilidad para así colocarlos en su AVA según las necesidades y planeación de los DI (Ver tabla 2)

\section{Tabla 2}

Categorización de Herramientas Web.

\begin{tabular}{|c|c|c|}
\hline Тіро & Actividad & $\begin{array}{l}\text { Herramienta } \\
\text { WEB }\end{array}$ \\
\hline Presentaciones & $\begin{array}{l}\text { Creación de diapositivas y } \\
\text { publicación }\end{array}$ & $\begin{array}{l}\text { Slide Share, } \\
\text { Calameo, } \\
\text { Issuu, Drive }\end{array}$ \\
\hline Esquemas & $\begin{array}{l}\text { Creación de mapas concep- } \\
\text { tuales, mentales, cuadros } \\
\text { sinópticos, líneas de tiempo }\end{array}$ & $\begin{array}{l}\text { Cacoo, } \\
\text { Cmaptools, } \\
\text { Lucidchart, } \\
\text { Dipity, Myti- } \\
\text { meline }\end{array}$ \\
\hline Documentos & $\begin{array}{l}\text { Creación de documentos } \\
\text { escritos on line }\end{array}$ & $\begin{array}{l}\text { Google Drive, } \\
\text { Skydrive }\end{array}$ \\
\hline Videos & $\begin{array}{l}\text { Creación o Publicación de } \\
\text { videos } \\
\text { Vídeo conferencias }\end{array}$ & $\begin{array}{l}\text { Wideo, You- } \\
\text { tube, Vimeo, } \\
\text { Animoto }\end{array}$ \\
\hline Comic & $\begin{array}{l}\text { Creación de historietas y } \\
\text { comic }\end{array}$ & $\begin{array}{l}\text { Pixton, Witty- } \\
\text { comics }\end{array}$ \\
\hline Comunicación & $\begin{array}{l}\text { Permite compartir y divulgar } \\
\text { la información }\end{array}$ & $\begin{array}{l}\text { Hangouts, } \\
\text { Skype, Foros }\end{array}$ \\
\hline
\end{tabular}

Fuente: Saza (2017).

En la Figura 4 se evidencia un curso diseñado bajo los parámetros del diseño instruccional $\mathrm{AD}$ DIE desde el enfoque praxeológico, en este curso se usó la estrategia pedagógica Webquest, además se estructuró el curso con banners titulados

- Actividades: donde se expuso las actividades a realizar.

- Recursos: contenidos, software y herramientas para realizar las actividades.

- Entregables: espacio para entregar las tareas, cada uno de los temas se esquematizaron de la misma forma.

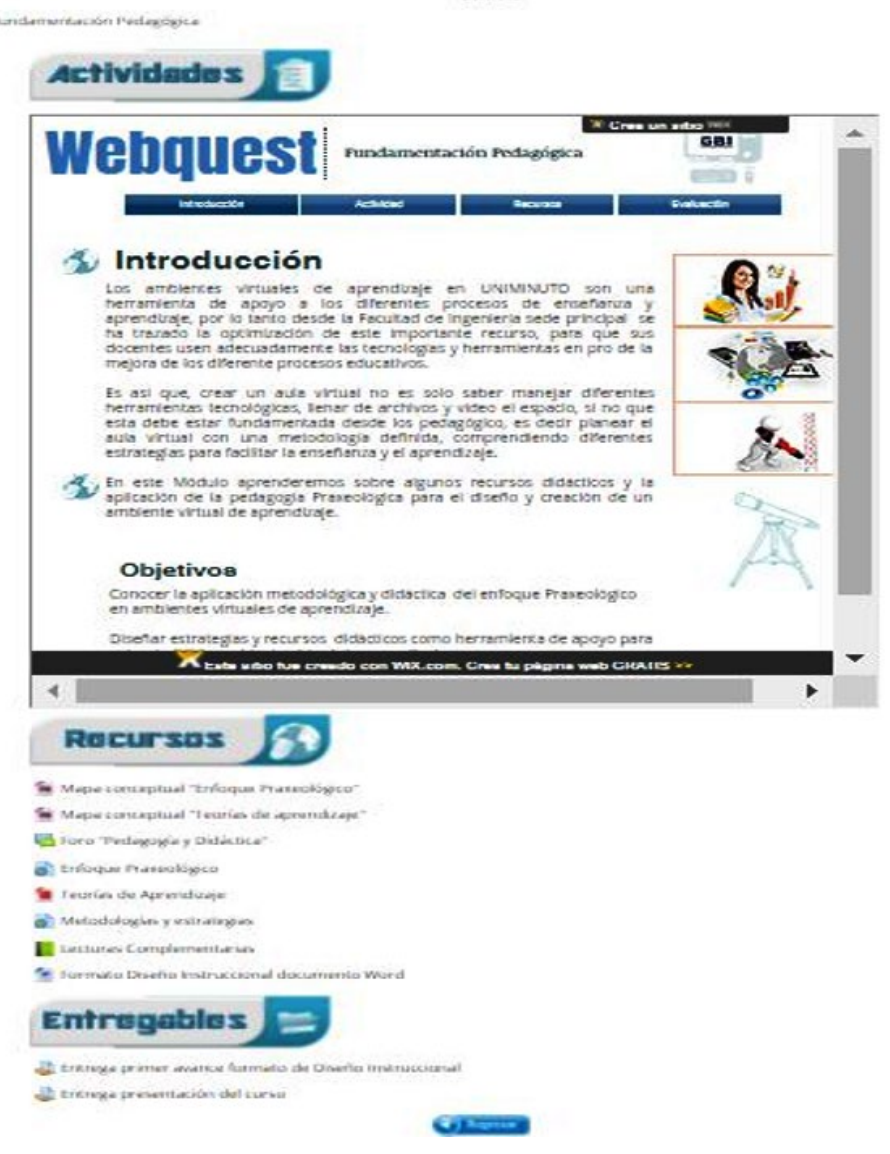

Figura 4. Curso creado bajo los parámetros del diseño instruccional modelo ADDIE. GBI (2017)

\section{Discusión y Conclusiones}

Con esta experiencia se puede concluir la necesidad de parametrizar un DI en todo UNIMINU$\mathrm{TO}$, y aconsejable que las instituciones educativas que están en el proceso de virtualización o uso de AVA cuenten con un modelo de DI, con el fin de crear un sello propio bajo la luz del enfoque pedagógico, con ello evitar la subutilización de las aulas virtuales, soslayando así, que se conviertan en repositorios o en el peor de los casos el no uso de este recurso tecnológico. 
Los docentes que desarrollaron el DI diseńaron y crearon aulas virtuales con sentido pedagógico claro, se evidencia la metodología y las didácticas, muestran variedad de recursos y actividades, en cuanto a los docentes que omitieron las actividades que tuvieron incidencia el DI, convirtieron sus espacios académicos como repositorios (solo archivos PDF con extensiones largas), no es claro qué metodología aplicar y en otros casos se convirtieron en espacios de foros para leer archivos.

La experiencia de capacitación en AVA partió de la aplicación de la pedagogía Praxeológica, enfoque que nace del ejercicio de la reflexión permanente, el cual permite articular diferentes procesos pedagógicos en los AVA; sin embargo, para los docentes no licenciados es complejo apropiar este ejercicio debido a su formación pragmática ya que ellos enfocan sus procesos de enseñanza más en el resultado que en el proceso.

En UNIMINUTO es necesario que, los docentes de las diferentes facultades tengan espacios de capacitación, donde converjan el uso de los AVA y la aplicación del enfoque praxeológico, para así crear cursos que respondan a las demandas educativas y las políticas de calidad de la universidad.

Esta experiencia puede ser un camino para otras instituciones educativas, en Colombia muchas universidades están ampliando su campo de oferta académica, creando programas académicos virtuales o virtualizando los existentes, como respuesta a las demandas económicas y sociales, sin embargo, existe la problemática de ¿Cuál es el camino a seguir para tener programas técnicos y profesiones con la calidad necesaria?, en este documento se socializa una de las tantas estrategias que puede servir para empezar el camino.

\section{REFERENCIAS BIBLIOGRÁFICAS}

Belloch, C. (2017). Diseño Instruccional. Unidad de Tecnología Educativa (UTE). Univer-sidad de Valencia. Recuperado de http://148.202.167.116:8080/xmlui/bitstream/handle/123456789/1321/EVA4.pdf?sequence $=1$ \&isAllowed $=y$

Benítez, M. (2010). El modelo de diseño instruccional Assure aplicado a la educación a distancia. Revista Tlatemoani, Académica de Investigación, 1. Recuperado de http://www. eumed.net/rev/tlatemoani/01/mgbl.pdf
Campos, A. (2009). Métodos mixtos de investigación: Integración de la investigación cuantitativa y la investigación cualitativa (Primera ed.). Bogotá: Editorial Magisterio.

Centeno, P. (2017). Una experiencia de estandarización utilizando el modelo ADDIE en la elaboración de guías temáticas. Revista e-Ciencias de la Información, 7 (1), 1-13. https://doi.org/10.15517/eci.v7i1.25755

Coll, C. \& Monereo, C. (2008). Psicología de la educación virtual. Madrid: Ediciones Morata, S. L. Estévez, A., Castro, J., \& Rodríguez, H. (2015). La educación virtual en Colombia: expo-sición de modelos de deserción Apertura. Revista Innovación Educativa, 7 (1), 1-10. Re-cuperado de https:// www.redalyc.org/pdf/688/68838021007.pdf

Juliao, C. G. (2002). La Praxeología: una teoría de la práctica. Bogotá: Uniminuto.

Juliao, C. G. (2011). El enfoque Praxeológico. Bogotá: Uniminuto.

Lopez, P. \& Fachelli, S. (2015). Metodología de la investigación social Cuantitativa. Barce-lona (España): editorial Universidad Autónoma de Barcelona.

Morales, B., Edel, R. \& Aguirre, G. (2014). Modelo ADDIE (análisis, diseño, desarrollo, implementación y evaluación): Su aplicación en ambientes educativos. Esquivel, I. Los Modelos Tecno-Educativos, revolucionando el aprendizaje del siglo XXI (pp 27 - 40). Mé-xico. Recuperado de https://www.uv.mx/personal/iesquivel/files/2015/03/los_ modelos_tecno_educativos_revolucionando_el_aprendizaje_del_siglo_xxi-4.pdf

Muñoz, P \& González, M (2009) El diseño de materiales de aprendizaje multimedia y las nuevas competencias del docente en contextos teleformativos. Editorial Bubok, Recupe-rado de https://books.google.com.co/ books?id=oegsuFHM5tMC\&pg=PA15\&dq=modelo+addie $\&$ hl $=$ es-419\&sa $=X \&$ redir_esc $=y \# v=$ onepage $\& \mathrm{q}=$ modelo\%20addie $\& \mathrm{f}=$ false

Otzen, T. \& Manterola, C. (2017). Técnicas de muestreo sobre una población a estudio. International Journal of Morphology, 35 (1), 227-232. https://doi.org/10.4067/S071795022017000100037

Pérez, J. R., López, R. F., \& Peralta, O. M. (2004). Hacia una educación de calidad: ges-tión, instrumentos y evaluación. 3a Edición. Madrid: Narcea.

https://doi.org/10.31619/caledu.n21.335

Sánchez. J, García, J., Juárez, Y., \& Sánchez, S. (2018). Diseño Instruccional ADDIE co-mo Metodología Pedagógica para la enseñanza-aprendizaje a través de Realidad Aumen-tada. En Prieto, M., Pech, S., Francesa, A. Tecnologías y Aprendizaje: Investigación y Práctica (pp 491-499) Editorial CIATA.org-UCLM Ciudad Real, España. Recuperado de https://www.researchgate.net/profile/Heidi_Angelica_Salinas_Padilla/publication/331177686_Facebook_meta-analisis_sobre_su_uso_educativo/links/5c6aedac92851c1c9dea8f23/Facebook-meta-analisis-sobre-su-uso-educativo. pdf\#page $=493$

Velarde, A., Dehesa, J., López, E., \& Márquez, J. (2017). 
Los vídeos tutoriales como apo-yo al proceso de enseñanza aprendizaje y sus implicaciones pedagógicas en el diseño instruccional. EDUCATECONCIENCIA, 14(15). Recuperado de http://tecnocientifica.com.mx/educateconciencia/ index.php/revistaeducate/article/view/330/307

Williams, Schrun, Sangrá \& Guardia (s.f). Modelos de diseño instruccional. OUC, (Univer-sitat Oberta de Catalunya). Recuperado de http://aulavirtualkamn.wikispaces.com/ file/view/2.+MODELOS+DE+DISE\%C3\%91O+INSTRUCCIONAL.pdf 